Clues to the laws of ecosystems

\author{
DavidJ. Beerling
}

Climate Change and Its Biological Consequences. By David M. Gates. Sinauer: 1993. Pp. 280. \$18.95, £16.95 (pbk).

Biotic Interactions and Global Changes. Edited by Peter M. Kareiva, Joel G. Kingsolver and Raymond B. Huey. Sinauer: 1993. Pp. 480. \$65, £58.95 (hbk); $\$ 32.95, £ 29.95$ (pbk).

ARE there fundamental laws governing the behaviour of ecosystems? If so, how long will it be before we can expect to discover them? Unfortunately, unlike physicists and chemists, ecologists have few, if any, general laws, and none that can be applied to the problem of predicting the responses of ecosystems to global climate change.

Ecologists are now trying to catch up with the physical sciences. The perceived threat of global change is forcing them to focus, in unprecedented detail, on how ecosystems function and interact with the environment. Simple correlations are just not sufficient - global change has redirected ecologists' attention towards finding generalities that are both precise and realistic.

D. M. Gates provides a readable and up-to-date summary of many of the aspects of global ecology required for an understanding of the present state of play. The coverage of topics is necessarily diverse, and brief, ranging from the astronomical theory of orbital forcing as a mechanism for Quaternary climatic change, to plant physiology, drought and El Niño events. Emphasis is placed on vegetation responses to global change; past vegetational response, the use of forest models and ecosystem responses comprise most of the book. Each topic is given a competent treatment that will be of use to students new to the subject but adds little to the knowledge of those already working in the field.

P. M. Kareiva and colleagues have edited a very different volume, pitched more at researchers. Concentrating only on ecosystem responses to global changes (excluding oceanic interactions and biogeochemical cycles), they have compiled chapters written by experts in their fields that attempt to develop new concepts rather than simply review the existing literature. The volume arose from a workshop on the effects of global change on populations and communities of plants and animals. In general, the approach works well. Of particular interest is the chapter by David Tilman on plant responses to carbon dioxide and that by Stephen Pacala and George
Hurt on the difficulties of forest models. Other chapters concentrate on stream communities, birds and interactions between plants and animals. An evolutionary section deals with species extinction caused by variation in the rates of climate change, migration and habitat fragmentation. Moreover, it tackles the more subtle concept of microevolution and the genetic variation of populations. My only criticism of the book as a whole is the parochial selection of authors: 40 out of the 45 are based in the United States.

Biotic Interactions and Global Changes emphasizes the search for generalities and provides several clear pointers and clues to where to go from here. The prospect of discovering the laws of ecology must now seem brighter thanks, ironically, to the prospect of a warmer world.

David J. Beerling is in the Department of Animal and Plant Sciences, University of Sheffield, Sheffield S10 2UQ, UK.

\section{Gravity waver}

\section{Eric G. Adelberger}

Gravitational Experiments in the Laboratory. By Y. T. Chen and Alan Cook. Cambridge University Press: 1993. Pp. 268. $£ 40, \$ 59.95$.

THE past decade has seen a small renaissance in laboratory tests of gravity, stimulated by a new way of interpreting the remarkably sensitive results of these ingenious mechanical experiments. The experiments are now viewed not so much as testing the theory of gravity (general relativity has passed all tests with flying colours, including those derived from the beautifully precise binary pulsar data) but as searches for possible new and very feeble interactions that may lie hidden 'underneath' gravity. There is a need for a new book aimed at those who seek an overview of the field or wish to understand the power and limitations of the techniques. Gravitational Experiments in the Laboratory will be useful to such readers, but is oddly flawed as well.

With commendable generality, Chen and Cook begin by discussing a linear oscillator in contact with a thermal bath, and then consider other possible disturbances of the oscillator. They apply this foundation to tests of the universality of free fall (the weak equivalence principle), to tests of the $1 / r^{2}$ law (the gravitational Gauss law) and finally to the notoriously difficult measurements of the newtonian gravitational constant $G$. (Although Boys claimed in 1889 that his torsion balance would be able to determine $G$ to a precision of one part in $10^{4}$, this goal has not yet been attained.)
The authors give a good account of the history of the field. But it has a curiously 'old-fashioned' flavour because it omits most of the developments since the reanalysis by $\mathrm{E}$. Fischbach and colleagues in 1986 of the classic Eötvös equivalence principle data, which seemed to provide evidence for a new fundamental interaction or 'fifth force'. The book also contains serious errors. For example, tests of the weak equivalence principle that compare the gravitational accelerations of different materials towards the Sun are explained as relying on tidal forces. This is wrong: these experiments do not probe tidal forces, which vary as $1 / r^{3}$, but the main gravitational force, which varies as $1 / r^{2}$. Another serious error concerns F. Witteborn and W. Fairbank's test of gravity using freely falling electrons. Their observation that electrons in the centre of a hollow conducting tube do not fall has never been confirmed. And L. Schiff and M. Barnhill's theoretical explanation for this 'fact', which Chen and Cook use as evidence that electrons feel the expected gravitational forces, is now thought to be in error.

There are also strange quirks in the discussion of experimental matters: circuit diagrams that make no sense and subsequent discussions that are therefore impenetrable; quantities given with many significant figures for no purpose; orderof-magnitude errors (a capacity of 0.5 picofarads is equated to that of 1 metre of coaxial cable); and crucial points misstated (in Mössbauer tests of the gravitational redshift, the temperatures of source and absorber have to be carefully controlled not because the first-order Doppler effect broadens the line but because the second-order effect shifts it).

A careful reader able to separate the wheat from the chaff will find much useful material in this book. However, a student wishing to learn about the field will need guidance on what not to accept, while someone wanting to know the status of the field will have to look elsewhere to learn about recent developments.

Eric G. Adelberger is in the Department of Physics, University of Washington, Seattle, Washington 98195, USA.

- Cambridge University Press has also just published a new edition of Theory and Experiment in Gravitational Physics by Clifford M. Will, price £19.95, \$37.95. For a review of the first edition, see Nature 299, 284; 1982

\section{Correction}

In his review of DNA on Trial (Nature 363, 122; 13 May 1993), John F. Y. Brookfield incorrectly attributes a chapter written by $P$. Rabinow to the editor of the volume, P. R. Billings. Our apologies for the error. 